\title{
Interference or experiment for \\ not: analysis of Young's a single-cycle pulse
}

\section{G. Girieud}

\section{S. F. Pereira}

s.f.pereira@.tudelft.nl
Delft University of Technology, Department of Imaging Science and Technology, Lorentzweg 1, 2628CJ Delft, The Netherlands

Delft University of Technology, Department of Imaging Science and Technology, Lorentzweg 1, 2628CJ Delft, The Netherlands

An analysis of Young's interference experiment for single cycle pulses showing the time evolution of the interference pattern due to two square apertures is obtained in the Fresnel regime. The fact that the diffraction pattern from each aperture is not constant in time has consequences on the interference pattern. We have also analyzed the changes in the spectrum at different regions at the observation plane. This position-dependent spectrum results in frequency beating of the interference pattern for the case of two apertures. [D0I: $10.2971 /$ jeos.2006.06016]

Keywords: Ultrafast phenomena, scalar diffraction, interference

\section{Introduction}

During the past decade, the technology of ultrashort pulse lasers has been rapidly developed and many applications of ultrashort pulses in areas such as communications, spectroscopy, chemistry, and microscopy are found. In these applications, it is important to understand the properties of ultrashort pulses on propagation [1]-[8].

In many cases, the diffraction pattern for continuous-wave illumination can be calculated using the Fresnel diffraction theory that describes the light propagation in the free space. However, calculations based on monochromatic continuouswave illumination are not valid for ultrashort pulses and we must consider the spectral width and shape of the pulses. As pointed out in Ref. [1], the diffraction of ultrashort pulses evolves in time and the diffraction pattern is very different from the one corresponding continuous-wave illumination. This effect is the consequence of the difference in propagation time of wavelets that originate from different points of the aperture to a point at the observation plane as a function of the wavelength.

In this article we analyze the evolution in time of the diffraction pattern of an ultrashort pulse through two square apertures in the Fresnel regime. The distance and size of the apertures are such that in the continuos-wave case, an interference pattern is observed as in the classical example of the Young's interference experiment. But as one can see here, in the case of an ultrashort pulse, the interference fringes are not constant in time; they have a temporal evolution as the pulse hits the aperture. By a proper choice of the parameters of the aperture size and distance to the observation plane, it is possible to find situations during the time evolution of the diffraction pattern where two separate spots (no interference) are observed or a classical interference pattern.
In the first part of this paper, we introduce the calculation of the diffraction pattern for a square aperture using the Fresnel expression. In a second part, we show the profile and the spectrum of the diffraction pattern through a square aperture as a function time using as an example pulses in terahertz regime. In third part, the time evolution of the interference pattern as well as the spectrum of the Young's interference fringes is shown.

\section{THEOR Y}

\subsection{Fresnel diffraction}

In order to do the first step of the diffraction pattern calculation of an ultrashort pulse we follow the lines of Ref. [9], i.e., we calculate the diffraction pattern for continuous wave illumination in the Fresnel approximation. The general geometry of the problem is shown in Figure 1.

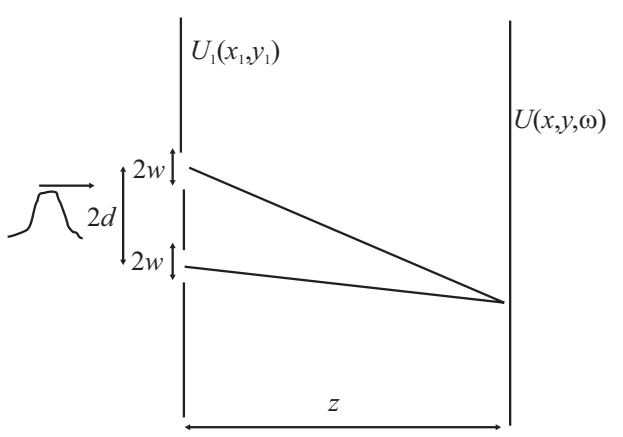

FIC. 1 Geometric configuration of the diffraction of one or two apertures: $2 w$ is the width of the slits, $2 d$ is the distance between the centres of the two slits, and $z$ is the distance between the aperture and observation planes. 
We suppose that a square aperture of width $2 w$ is illuminated by a plane wave of unit amplitude. The distribution of the complex field immediately behind the aperture is given by

$$
U_{1}\left(x_{1}, y_{1}\right)=\operatorname{rect}\left(\frac{x_{1}}{2 w}\right) \operatorname{rect}\left(\frac{y_{1}}{2 w}\right)
$$

where $x_{1}$ and $y_{1}$ are the coordinates in the plane of aperture and $\operatorname{rect}(\mathrm{x})$ is the rectangular function. With the Fresnel diffraction equation, the distribution of the complex field $U(x, y, \omega)$ at the observation plane located at a distance $z$ from the aperture and for the incident plane with distribution $U_{1}\left(x_{1}, y_{1}\right)$ is given by:

$$
\begin{array}{r}
U(x, y, \omega)=\frac{e^{i k z}}{i \lambda z} \int_{-w}^{+w} \exp \left[i \frac { k } { 2 z } \left[\left(x-x_{1}\right)^{2}+\right.\right. \\
\left.\left.\left(y-y_{1}\right)^{2}\right]\right] d x_{1} d y_{1}
\end{array}
$$

where $x$ and $y$ are the coordinates in the observation plane, $\lambda=2 \pi \mathrm{c} / \omega$ is wavelength of the incident field and $k$ is the wavevector.

\subsection{Spectrum of the pulse}

The second step of the calculation is described in Refs. [1, 2]. The illumination of an ultrashort laser pulse is not monochromatic but includes a spectral distribution. Thus, the field in the observation plane is a coherent superposition of the distributions from each individual frequency component with a certain amplitude distribution. The Fresnel equation (2) becomes:

$$
\begin{array}{r}
U(x, y, \omega)=V(\Delta \omega) \frac{e^{i \cdot k \cdot z}}{i \lambda z} \iint_{-w}^{+w} \exp \left[i \frac { k } { 2 z } \left[\left(x-x_{1}\right)^{2}+\right.\right. \\
\left.\left.\left(y-y_{1}\right)^{2}\right]\right] d x_{1} d y_{1}
\end{array}
$$

where $\Delta \omega=\omega-\omega_{0}$ and $V(\Delta \omega)$ is the spectrum of the ultrashort laser pulse given by [3, 4]:

$$
V(\Delta \omega)=\left[\frac{s+1}{\omega_{1}}\right] \frac{\left(\omega-\omega_{0}\right)^{s}}{\Gamma(s+1)} \exp \left[-(s+1) \frac{\omega-\omega_{0}}{\omega_{1}}\right],
$$

if $\omega \geq \omega_{0}$ and 0 , else, with $\omega_{0}$ the frequency shift, $\omega_{1}$ the spectrum width and $s$ is a positive parameter (small values of $s$ correspond to very short pulses in the time domain). The value of the frequency shift $\omega_{0}$ gives an estimate of the central wavelength of the spectrum. Eq.(3) can be rewritten as:

$$
U(x, y, \omega)=V(\Delta \omega) \frac{e^{i k z}}{i} I(x, \omega) I(y, \omega)
$$

where

$$
\begin{aligned}
& I(x, \omega)=\frac{1}{\sqrt{\lambda} z} \int_{-w}^{+w} \exp \left[i \frac{\pi}{\lambda z}\left(x-x_{1}\right)^{2}\right] d x_{1} \\
& I(y, \omega)=\frac{1}{\sqrt{\lambda} z} \int_{-w}^{+w} \exp \left[i \frac{\pi}{\lambda z}\left(y-y_{1}\right)^{2}\right] d y_{1}
\end{aligned}
$$

The integrals $I(x, \omega)$ and $I(y, \omega)$ are related to Fresnel integrals $C(z)$ and $S(z)$ as described in detail in Ref. [8]:

$$
\begin{aligned}
& I(x, \omega)=\frac{1}{\sqrt{2}}\left\{\left[C\left(\alpha_{2}(\omega)\right)-C\left(\alpha_{1}(\omega)\right)\right]+\right. \\
& \left.i\left[S\left(\alpha_{2}(\omega)\right)-S\left(\alpha_{1}(\omega)\right)\right]\right\} \\
& I(y, \omega)=\frac{1}{\sqrt{2}}\left\{\left[C\left(\beta_{2}(\omega)\right)-C\left(\beta_{1}(\omega)\right)\right]+\right. \\
& \left.i\left[S\left(\beta_{2}(\omega)\right)-S\left(\beta_{1}(\omega)\right)\right]\right\}
\end{aligned}
$$

With this substitution, equation (4) becomes:

$$
\begin{gathered}
U(x, y, \omega)=V(\Delta \omega) \frac{e^{i \frac{\omega}{c} z}}{i}\left\{\left[C\left(\alpha_{2}(\omega)\right)-C\left(\alpha_{1}(\omega)\right)\right]+\right. \\
\left.i\left[S\left(\alpha_{2}(\omega)\right)-S\left(\alpha_{1}(\omega)\right)\right]\right\}\left\{\left[C\left(\beta_{2}(\omega)\right)-C\left(\beta_{1}(\omega)\right)\right]+\right. \\
\left.i\left[S\left(\beta_{2}(\omega)\right)-S\left(\beta_{1}(\omega)\right)\right]\right\}
\end{gathered}
$$

This equation describes the spectrum of the diffracted field at the observation plane.

\subsection{Time dependence}

The diffracted field as a function of time $U(x, y, t)$ of an ultrashort pulse is given by the inverse Fourier transform of Eq.(5):

$$
\begin{gathered}
U(x, y, t)=\int_{-\infty}^{+\infty} U(x, y, \omega) \cdot e^{-i \omega t} d \omega \\
U(x, y, t)=\int_{\omega_{0}}^{+\infty} V(\Delta \omega) \frac{e^{i \frac{\omega}{c} z}}{i}\left\{\left[C\left(\alpha_{2}(\omega)\right)-C\left(\alpha_{1}(\omega)\right)\right]+\right. \\
\left.i\left[S\left(\alpha_{2}(\omega)\right)-S\left(\alpha_{1}(\omega)\right)\right]\right\}\left\{\left[C\left(\beta_{2}(\omega)\right)-C\left(\beta_{1}(\omega)\right)\right]+\right. \\
\left.i\left[S\left(\beta_{2}(\omega)\right)-S\left(\beta_{1}(\omega)\right)\right]\right\} e^{-i \omega t} d \omega
\end{gathered}
$$

Next, we show the numerical computation of the diffraction integral of the time evolution as well as the spectrum at the observation planes for two situations: single and double square apertures.

\section{S INGLE APERTURE}

\subsection{Time dependence of the diffrac- tion pattern}

In this section we present simulations of the temporal evolution of the diffraction pattern from a square aperture in the Fresnel regime.

We have chosen to do our calculations in the terahertz domain since in this frequency regime ultrashort pulses containing one or two cycles are routinely being produced in laboratories. We consider a spectrum width $\omega_{1}=6.10^{12} \mathrm{rad} / \mathrm{s}$, which corresponds to a pulse whose duration is about $1 \mathrm{ps}$, the frequency shift $\omega_{0}=10^{14} \mathrm{rad} / \mathrm{s}$ (wavelength of $20 \mu \mathrm{m}$ ), and the parameter $s$ is set to 1 . Here we show an example where the time evolution of the diffraction pattern can be clearly seen. The choice of the size of the apertures and the distance between diffraction and observation planes is such that the time 
delay due to the path difference between the diffracted light at the centre and at the edge of the main lobe is larger than the temporal width of the light pulse. In the example here, the aperture width is $w=0.1 \mathrm{~mm}$ and the distance between the aperture and observation planes $z$ is $0.250 \mathrm{~m}$. With these parameters, the Fresnel number $N_{0}=\omega_{0} w^{2} /(2 \pi c z)$ is 0.002 . The data is shown in the interval of $x=[-20 \mathrm{~mm}, 20 \mathrm{~mm}]$. In Figure 2 we plot the $2 \mathrm{D}$ diffraction pattern (left side) and the corresponding profile at the $x$ axis (right side) of the central lobe for various time shots.
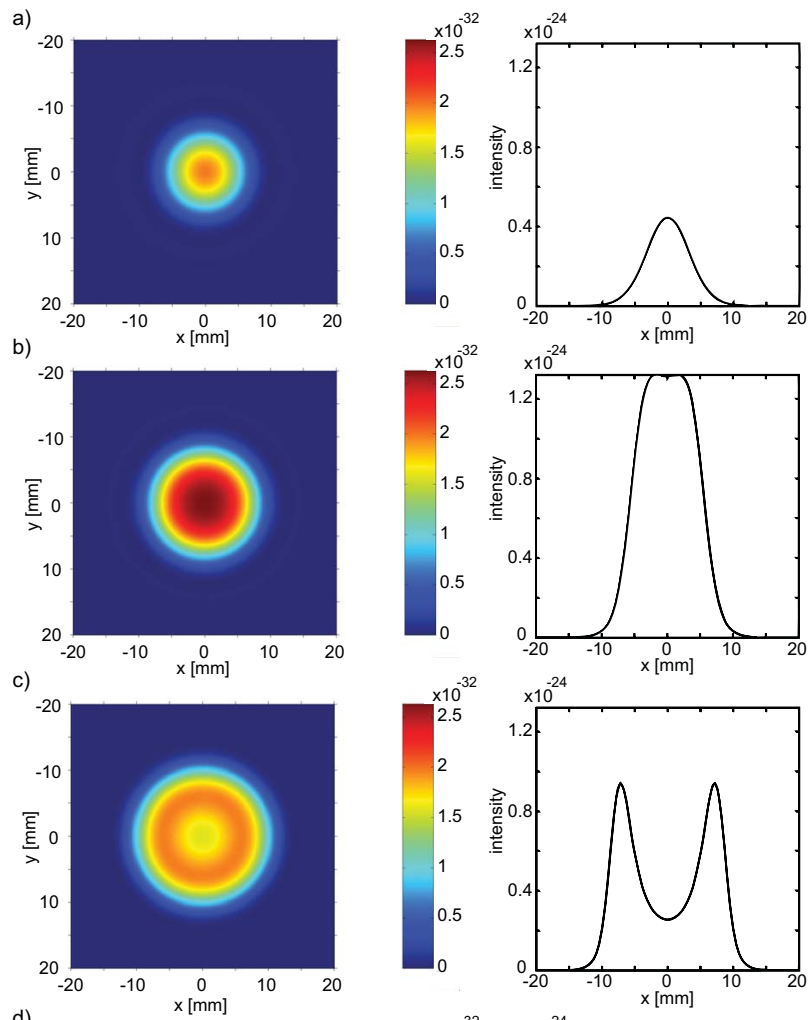

d)
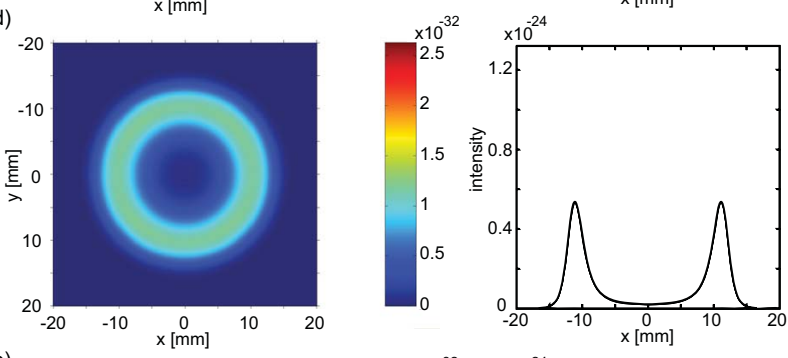

e)
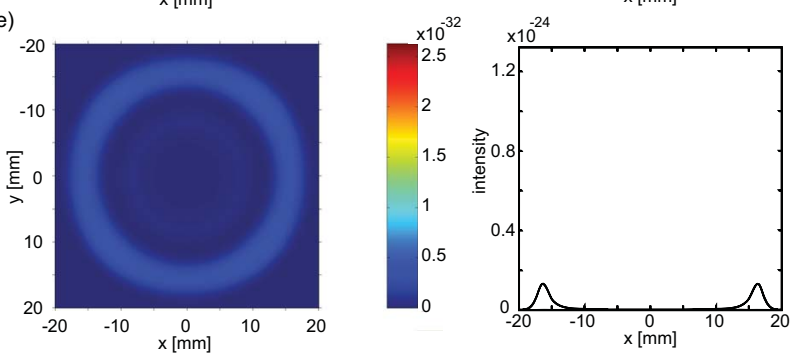

In Figure 3 we show the superposition of a few profiles of the time shots of Figure 2 for a wider range of values of the $x$ axis $(-0.1$ to $0.1 \mathrm{~m})$ in order to show how the diffraction pattern spreads out further at the observation plane as the time evolves. The three peaks at the central area appear first (early times) while the side lobes appear later. Note also the spliting of the central peak as time evolves. If plotted in $2 \mathrm{D}$, the diffraction figure follows the one from a square aperture, i.e., a central area and side lobes distributed in two perpendicular lines.

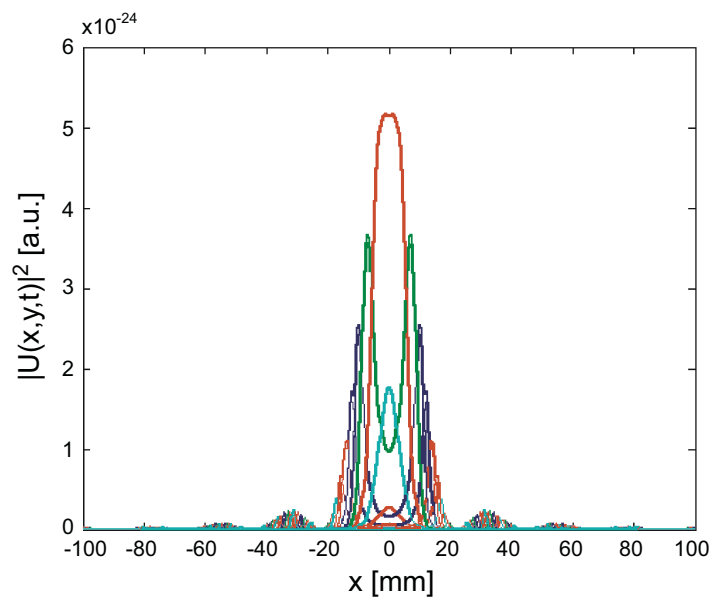

FIG. 3 Superposition of the profiles at different time shots of the diffraction pattern due to a single aperture along the $x$ axis. The parameters are the same that of Figure 2 except that the $x$-axis here has been extended to beyond the central region of the diffraction pattern so that the side lobes can be seen.

\subsection{Spectrum of the diffraction pattern}

Apart from the evolution of the diffraction pattern, the spectral distribution of the ultrashort pulse implies also consequences on the spectral distribution of the diffraction pattern, as can be seen from the frequency dependence on Eq.(6). The spectrum at different points on the observation plane has a blue or red shift with respect to the original spectrum at the aperture plane [10]. In Table 1 we quantify the shift in the peak of the spectrum for various $x$-values at the aperture plane with positive (negative) values representing shifts in the spectrum towards lower (higher) frequencies.

\begin{tabular}{|l||l|}
\hline$x(m)$ & $\Delta \omega\left(10^{14} \mathrm{rad} / \mathrm{s}\right)$ \\
\hline 0 & 0.0014 \\
\hline 0.0025 & 0.0013 \\
\hline 0.005 & 0.0010 \\
\hline 0.0075 & 0.0006 \\
\hline 0.01 & 0.00001 \\
\hline 0.0125 & -0.0009 \\
\hline 0.015 & -0.0023 \\
\hline 0.02 & -0.0085 \\
\hline
\end{tabular}
time that $|U(x, y, t)|^{2}$ is maximum at the observation plane. The spectrum width is $6.10^{12} \mathrm{rad} / \mathrm{s}$, the frequency shift $10^{14} \mathrm{rad} / \mathrm{s}$, the aperture width is $w=0.1 \mathrm{~mm}$ and the distance $z$ is $0.250 \mathrm{~m}$. With these parameters, the Fresnel number $N_{0}$ is 0.002 . Right: for each $2 \mathrm{D}$ pattern (left side) we plot the corresponding $1 \mathrm{D}$ profile at $\mathrm{y}=0$. Intensity units are arbitrary.
FIG. 2 Left: Time evolution of the cental lobe of the diffraction pattern in the $x y$ plane for $x, y$ ranging from -20 to $20 \mathrm{~mm}$ of the diffraction pattern of a ultrashort pulse
TABLE 1 Shift in the spectrum $\Delta \omega$ with respect to the original spectrum (i.e., spectrum at the aperture plane) at various positions $x$ at the observation plane. 


\section{T W 0 APERT URES}

\subsection{Time dependence of the inter- ference pattern}

Having seen how the diffraction pattern from one aperture evolves, we analyse further the Young's interference experiment by considering two square apertures separated by the distance $2 d$. We calculate the diffraction pattern for the same parameters as for one aperture.

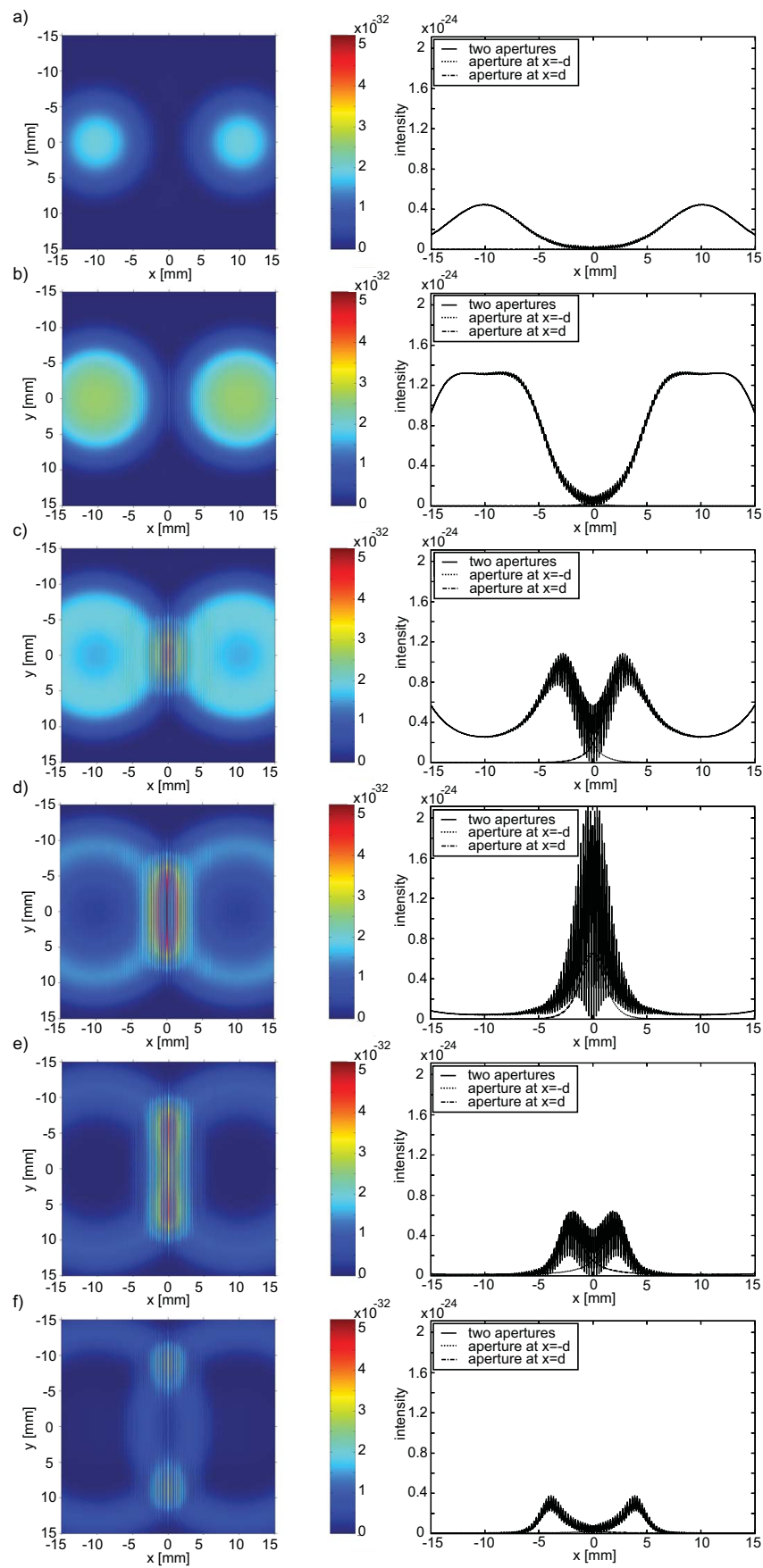

FIG. 4 Time evolution of the Young's interference experiment for a single ultrashort pulse in the $x y$ plane with $x, y$ ranging -15 to $15 \mathrm{~mm}$ at the following times from (a) to $(f):-0.3,0,0.3,0.7,1$ and $1.3 \mathrm{ps}$. The spectrum width is $6.10^{12} \mathrm{rad} / \mathrm{s}$, the frequency shift $10^{14} \mathrm{rad} / \mathrm{s}$, the aperture width is $w=0.1 \mathrm{~mm}$, the distance $z$ is $0.250 \mathrm{~m}$ and the distance between the apertures is $2 d=2.0 \mathrm{~cm}$. With these parameters, the Fresnel number $\mathrm{N}_{0}$ is 0.002 . For each $2 \mathrm{D}$ pattern the profiles in the $x$ axis are shown. The dashed lines are the diffraction pattern due one aperture.
The theoretical aspect of this problem is not very different than for one aperture; it is just the sum of the diffraction patterns from each aperture. In this case, the Fresnel equation becomes:

$$
\begin{gathered}
U(x, y, \omega)=V(\Delta \omega) \frac{e^{i k z}}{i \lambda z}\left\{\int _ { - d + 2 w } ^ { - d } \int _ { - w } ^ { + w } \operatorname { e x p } \left[i \frac { k } { 2 z } \left[\left(x-x_{1}\right)^{2}+\right.\right.\right. \\
\left.\left.\left(y-y_{1}\right)^{2}\right]\right] d x_{1} d y_{1} \int_{d}^{d+2 w+w} \int_{-w}^{+w} \exp \left[i \frac { k } { 2 z } \left[\left(x-x_{1}\right)^{2}+\right.\right. \\
\left.\left.\left(y-y_{1}\right)^{2}\right] d x_{1} d y_{1}\right\}
\end{gathered}
$$

In Figure 4, we show the evolution in time of the diffraction pattern due to two apertures in the $x-y$ plane and the crosssections at $\mathrm{y}=0$ ( $x$ axis). The parameters for the incident light pulse and the diameter of the individual aperture are the same as in Figures 2. The distance between the two apertures is $2 d=$ $2.0 \mathrm{~cm}$ and the diffraction pattern is shown at the observation interval $x=[-1.5,1.5 \mathrm{~cm}]$. From this example, we see that the obtained results agree with the argument given at the beginning of this paper, i.e., that the interference fringes evolve in time. We notice that at the early times there is no interference, because the diffracted pattern due to each aperture is concentrated at a small region. As the diffracted patterns spread out, clear interference fringes with high visibility between the inner sides of the rings occur. The interference fringes can better be appreciated by looking at the profiles at the $x$ axis from Figure 4.

\subsection{Spectrum of the interference pattern}

In the previous section we showed that the spectral distribution of the light pulse was not the same at every point on the observation plane but shifted with respect to the spectrum of the laser pulse at the aperture plane, with the shift being either towards higher or lower frequencies. This shift has consequences in the resulting spectra due to two apertures, since frequency beating will occur in some positions of the observation plane. On Figure 5 , we show the spectra of the light pulse at different points at the observation plane.

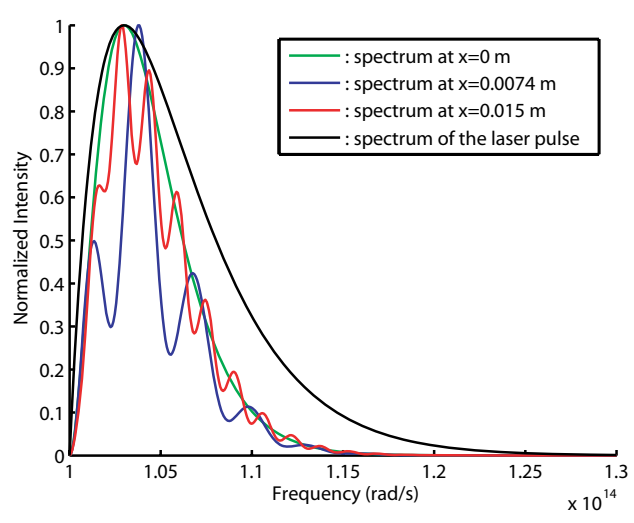

FIG. 5 Normalised spectral distribution due to the diffraction of two apertures at the following points of the observation plane at $x=0,0.0074$ and $0.015 \mathrm{~m}$. Tha black solid line represents the spectrum of the pulse before the aperture. The parameters are the same as in Figure 4 
We notice that the spectral distribution at the centre has no interference fringes, since the spectral shift due to each aperture is symmetrical at this point. At other positions, fringes will appear since the spectra due to each aperture are shifted with respect to each other. The frequency of the interference fringes increase for a point far from the centre, since the difference in frequency between the spectra also increases.

\section{C O N CLUSION}

By analysing the diffraction through a square aperture and Young's interference experiment for ultrashort pulses, we have demonstrated that the diffraction pattern and the interference fringes are not constant in time, as in the case of continuous wave illumination, but evolve as the laser pulse hits the apertures. We have also calculated the spectral distribution of the light at different regions at the observation plane and as result we see that spectral shifts towards higher or lower frequencies occur. As consequence, frequency beating in the case of interference of two apertures will take place.

Finally, we would like to emphasize the importance of looking at the evolution of the diffraction of ultrashort pulses. As seen in Figure 2 and 3, at earlier times of the propagation of the pulse through the aperture, the diffracted pattern is narrower than the integrated intensity distribution of the entire pulse. This means that a better resolution could be achieved in ultrashort pulse applications if one could record the diffracted light pulse only at earlier times. Further, we have shown that this concentrated intensity distribution has also consequences in the case of diffraction due to two apertures, as seen in Figures 4 . By choosing the time interval at earlier times (such as in Figures $4 \mathrm{a}$ and b) one can clearly see two separate spots at the observation plane (no interference), while at later times (Figures 4c-e) interference fringes with large visibility are obtained. In this way we conclude that whether interference of ultrashort pulses at the observation plane occurs or not depends on the time of the observation. The later remark could imply interesting interpretations of the phenomenon of interference and its link to time of observation.

\section{ACK N O W L E D E M E N T S}

The authors wish to acknowledge Joseph Braat for suggestions and comments on this paper.

\section{References}

[1] M. Lefrançois and S. F. Pereira, "Time evolution of the diffraction pattern of an ultrashort laser pulse" Opt. Expr. 11, 1114 (2003).

[2] M. Gu and X. S. Gan, "Fresnel diffraction by a circular and serrated apertures illuminated with an ultrashort pulsed-laser beam" J. Opt. Soc. Am. A 13, 771 (1996).

[3] C. J. R. Sheppard, "Bessel pulse beams and focus wave modes" J. Opt. Soc. Am. A 18, 2594 (2001).

[4] C. F. R. Caron and R. M. Potvliege, "Free-space propagation of ultrashort pulses: space-time couplings in Gaussian pulse beams" J. Mod. Opt. 46, 1881 (1999).

[5] Z. Jiang, R. Jacquemin, and W. Eberhardt, "Time dependence of Fresnel diffraction of ultrashort laser pulses by a circular aperture" Appl. Opt. 36, 4358 (1997).

[6] Z. Wang, Z. Zhang, Z. Xu, Q. Lin, "Spectral and temporal properties of ultrashort light pulse in far zone" Opt. Commun. 123, 5 (1996).

[7] M. Kline and I. W. Kay, Electromagnetic Theory and Geometrical Optics (Interscience Publs., a division of John Wiley a Sons, NY, 1965).

[8] L. Sereda, A. Ferrari, and M. Bertolotti, "Diffraction of a pulsed plane wave from an amplitude diffraction grating" J. Mod. Opt. 44, 1321 (1997),

[9] Joseph W. Goodman, Introduction to Fourier Optics (McGraw-Hill, NY, 1968).

[10] S. P. Veetil, N. K. Viswanathan, C. Vijayan, and F. Wyrowski, "Spectral and temporal evolutions of ultrashort pulses diffracted through a slit near phase singularities" Appl. Phys. Lett. 89, 041119 (2006). 